\title{
Preparation and Characterization of Monodisperse Polyacrylamide Microgels
}

\author{
Kong Soo KIM, Suk Hyeong CHO, and Jae Sup SHIN* \\ Department of Industrial and Engineering Chemistry, Chungbuk National University, \\ Cheongju 360-763, Korea \\ * Department of Chemistry, Chungbuk National University, \\ Cheongju 360-763, Korea
}

(Received October 17, 1994)

\begin{abstract}
Monodisperse microgels were prepared by the precipitation polymerization of acrylamide with acryloyl-terminated-poly(ethylene glycol) (PEG) macromonomer as a stabilizer. The process was carried in ethanol, methanol, and a mixture of two. The size of microgels was controlled to the range of $0.06-1.0 \mu \mathrm{m}$, changing the solvent. GPC profiles and particle size distribution of the microgels were narrow and monodisperse. Microgels were dispersable in water, and showed very low viscosity, and were stable in polar solvents and $\mathrm{KCl}$ aqueous solution.

KEY WORDS Macromonomer / Monodisperse / Precipitation Polymerization / Microgel / Polyacrylamide /
\end{abstract}

Polymer microgels are used in many fields as agents of paint, surface coating, and adhesive $^{1-3}$ and also biological and biomedical fields ${ }^{4-6}$ because microgels due to their low viscosity and specific surface area.

Emulsion polymerization and dispersion polymerization are methods for preparation of polymer particles having micron size and polydisperse particle size distribution. ${ }^{7,8}$ Polymer particles of monidisperse and submicron size were prepared by precipitation polymerization using methacrylic acid as a stabilizer. ${ }^{9}$

In this study, Polyacrylamide microgels were prepared by precipitation polymerization ${ }^{10}$ using macromonomer. The effects of solvent on the particle size of microgels in polymerization were determined. The shape and size of microgels were observed by electron microscopy, gel permeation chromatography (GPC), and autosizer.

Viscosity was measured by viscometer and the stability was determined by UV/Vis spectroscopy.

\section{EXPERIMENTAL}

\section{Materials}

Acrylamide (AAm) was recrystallized from acetone. $N, N^{\prime}$-methylene-bisacrylamide (MBAm) (Aldrich Chemical Co.) was recrystallized from methanol, and acryloyl terminated poly(ethyene glycol) macromonomer (AtPEGM) was synthesized according to a previous method. ${ }^{10}$ Benzoyl peroxide (BPO) was used without further purification. Ethanol, methanol, and dimethylformamide (DMF) were distilled before use.

\section{Precipitation Polymerization}

Given amounts of AtPEGM, AAm, MBAm and BPO were dissolved in $50 \mathrm{ml}$ alcoholic solvents. Polymerization was carried out at $60^{\circ} \mathrm{C}$ for $6 \mathrm{~h}$ under nitrogen atmosphere by adding $4.0 \times 10^{-3} \mathrm{moll}^{-1}$ of BPO initiator. The product was collected by centrifugation. The microgels were washed with methanol and diethylether three times and dried at room temperature under vacuum. 


\section{Measurements}

The size distribution of polymer particles was measured using electron micrographs and a Malvern Autosizer.

The shape of polymer particles was observed using scanning electron micrograph (SEM) (Hitachi S-530) and transmission electron micrograph (TEM) (Hitachi H-600).

Intrinsic viscosity of polyacrylamide microgels in various solvents was measured by an Ostwald viscometer. GPC chromatography (Waters 846) of the microgels was carried out (flow rate: $1 \mathrm{ml} \mathrm{min}^{-1}$; column Styragel 1000 and Styragel 500; temperature, room temperature; mobil phase, DMF). Stability was measured by UV/Vis spectroscopy (Kontron Uvicon 860) in water and other solvents.

\section{RESULTS AND DISCUSSION}

The polymer microgels were prepared according to precipitation polymerization reported earlier. ${ }^{10}$ In order to prepare monodisperse microgels, the stabilizer must be used in precipitation polymerization. ${ }^{11}$ In this system, the polyacrylamide microgels were obtained by steric stabilization of AtPEGM, as shown in the scanning electron micrographs (SEM) of Figure 1. Monodisperse microgels were formed when the AtPEGM was used as a stabilizer but coagulum was yielded when stabilizer was not used since aggregation between particles was protected by repulsion force and solvation layer of the poly(ethylene glycol) (PEG) part when polymer particles were closed.

Particle size was controlled by changing the concentrations of AAm, MBAm, and AtPEGM as previously reported, ${ }^{10}$ and size measured SEM and Malvern Autosizer was $0.1-$ $1.0 \mu \mathrm{m}$.

Figure 2 presents transmission electron micrographs (TEM) of the microgels. In order to them, the microgels were dispersed in water, and a drop of $\mathrm{OsO}_{4}(10 \mathrm{wt} \%$ aq.) was added. A drop of the stained microgel solution was placed on a copper grid coated with carbon

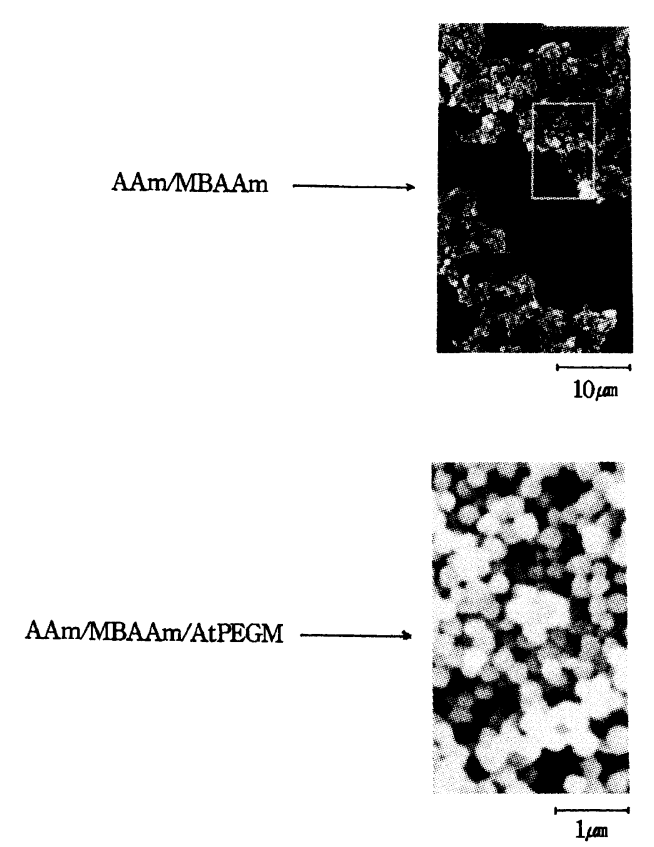

Figure 1. Scanning electron micrographs of particles prepared by precipitation polymerization in ethanol.

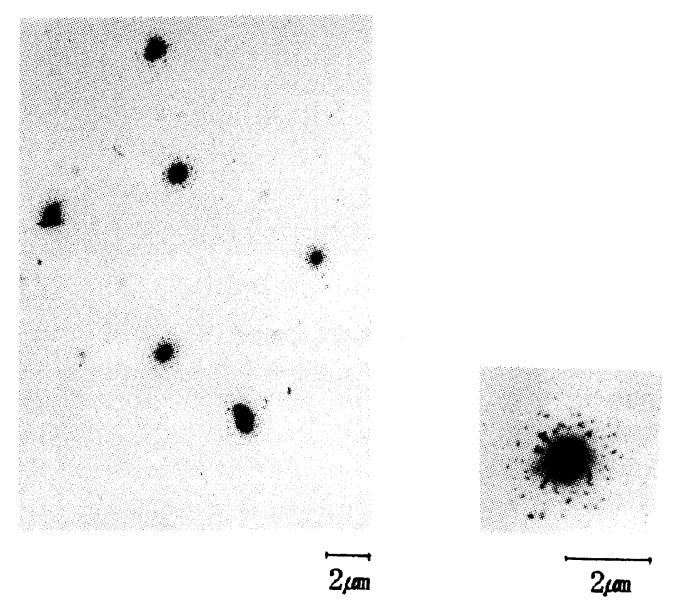

Figure 2. Transmission electron micrographs of polyacrylamide microgels.

film, and then dried gradually at $25^{\circ} \mathrm{C}$. As shown in Figure 2, microgels had spherical shape.

Table I and Figure 3 are molecular weight and distribution of microgels measured by GPC in $0.5 \mathrm{wt} \%$ of DMF solution. The elution 
Table I. Characterization of polyacrylamide microgels ${ }^{\mathbf{a}}$

\begin{tabular}{|c|c|c|c|c|c|c|c|c|}
\hline \multicolumn{4}{|c|}{ Feed $/ \times 10^{-2} \mathrm{moll}^{-1}$} & \multirow{2}{*}{$\frac{\left(D^{3}\right)^{1 / 3}}{n m}$} & \multirow{2}{*}{$\frac{[\eta]^{\mathrm{b}}}{\mathrm{g} \mathrm{dl}^{-1}}$} & \multirow{2}{*}{$M_{w} \times 10^{-9 c}$} & \multirow{2}{*}{$M_{w} / M_{n}^{\mathrm{d}}$} & \multirow{2}{*}{$\frac{\text { Yield }}{\%}$} \\
\hline AtPEGM & AAm & MBAAm & $\mathrm{H}_{2} \mathrm{O}$ & & & & & \\
\hline 0.6 & 15.4 & 0.8 & 0.0 & 192 & 1.30 & 5.3 & 1.02 & 75.8 \\
\hline 0.6 & 15.4 & 0.8 & 5.5 & 180 & 1.22 & 4.4 & 1.05 & 73.0 \\
\hline 0.6 & 15.4 & 0.8 & 11.0 & 173 & 1.11 & 3.8 & 1.02 & 73.5 \\
\hline 0.6 & 15.4 & 0.8 & 16.5 & 160 & 0.75 & 3.2 & 1.04 & 71.4 \\
\hline
\end{tabular}

${ }^{\mathrm{a}}[\mathrm{BPO}]=4.0 \times 10^{-3} \mathrm{moll}^{-1} \cdot{ }^{\mathrm{b}}$ In DMF at $25^{\circ} \mathrm{C}$. ${ }^{\mathrm{c}}$ Calculated by universal calibration curve. ${ }^{\mathrm{d}}$ Calculated by GPC profiles.

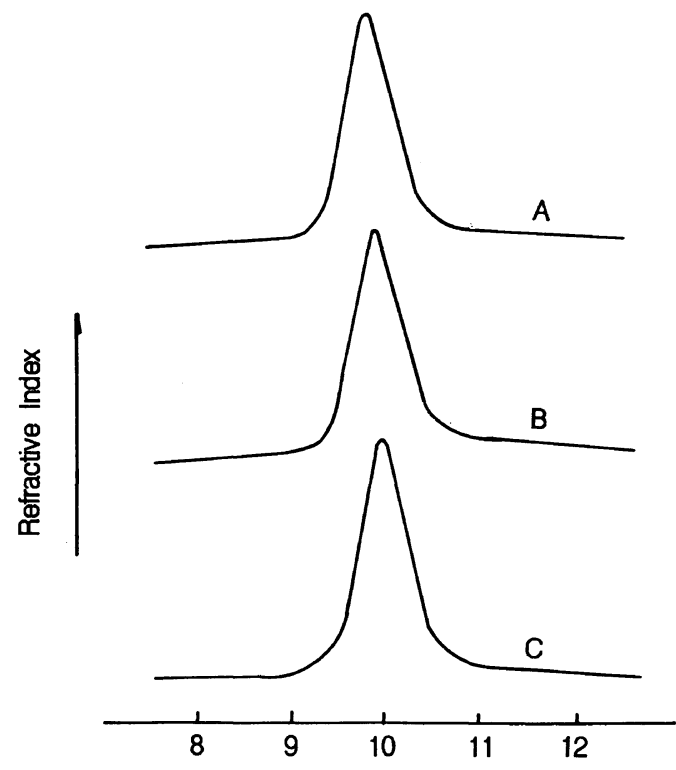

Eluent Count

Figure 3. GPC profiles of polyacrylamide microgels polymerized in ethanol containing water. (AtPEGM/ AAm $/ \mathrm{MBAm} / \mathrm{H}_{2} \mathrm{O} \times 10^{2} \mathrm{moll}^{-1}=\mathrm{A}, 0.6 / 15.4 / 4 / 0 ; \mathrm{B}, 0.6$ / 15.4/4/5.5; C, 0.6/15.4/4/11).

behavior of homopolymers and copolymers in GPC is governed by hydrodynamic volume. The 'universal' calibration curve of the product of intrinsic viscosity and molecular weight $([\eta] M)$ against elution volume can be applied to highly branched polymers. ${ }^{12}$ The introduction of the radius of the equivalent hydrodynamic sphere $\left(R_{\eta}\right)$ yields:

$$
[\eta] M=2.5\left(4 / 3 \pi R_{\eta}{ }^{3}\right) N_{\mathrm{A}}=2.5 V_{\eta} N_{\mathrm{A}}
$$

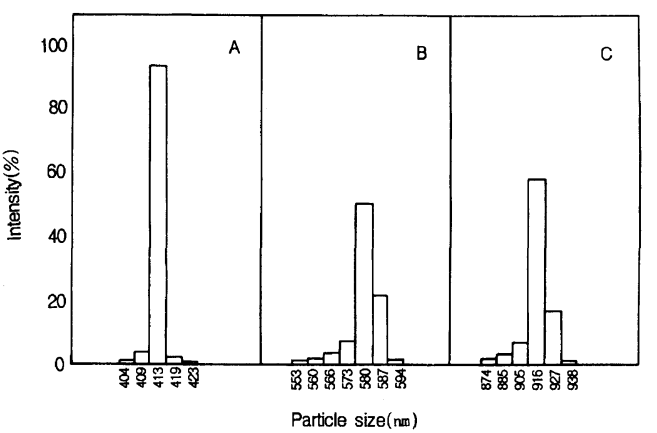

Figure 4. Particle size distribution of polyacrylamide microgels polymerized in ethanol containing water. (AtPEGM/AAm/MBAm $/ \mathrm{H}_{2} \mathrm{O} \times 10^{2} \mathrm{moll}^{-1}=\mathrm{A}, 0.6 /$ $15.4 / 4 / 5.5 ; \mathrm{B}, 0.6 / 15.4 / 4 / 11 ; \mathrm{C}, 0.6 / 15.4 / 4 / 16.5$ ).

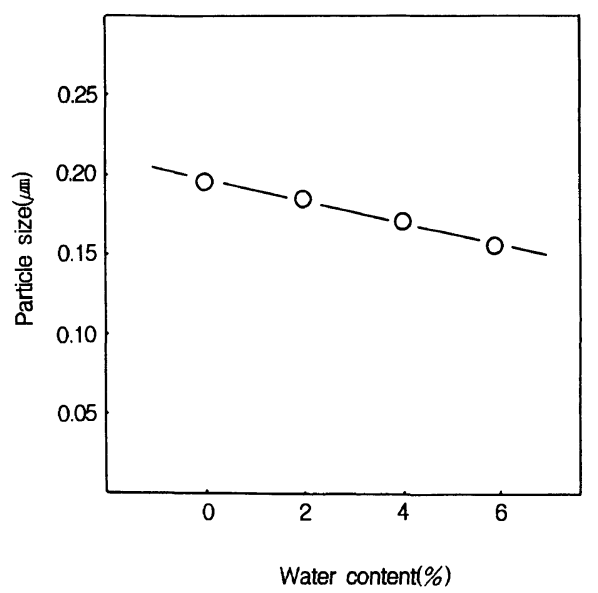

Figure 5. Influence of water content on particle size of polyacrylamide microgels (AtPEGM/AAm/MBAm $\times$ $\left.10^{2} \mathrm{moll}^{-1}=0.6 / 15.4 / 4\right)$.

where $N_{\mathrm{A}}$ is Avogadro's number and $V_{\eta}$ is the hydrodynamic volume. Molecular weights $\left(M_{w}\right)$ and molecular weight ratios $M_{w} / M_{n}$ are 
listed in Table I. As shown in Table I and Figure 3, microgels had narrow molecular weight distribution of $M_{w} / M_{n}=1.02-1.05$. Their average molecular weights calculated by universal calibration curve were very large as shown in Table I.

The microgels had monodisperse particle size distribution of $D_{w} / D_{n}=1.01-1.06$ as shown in Figure 4 also.

The effects of solvents on the size of microgels are shown in Figures 5, 6, and Table II. With increasing water in ethanol, particle size decreased. Higher solvency of the stabilizer moiety increased steric repulsive force between the nuclei, which might increase the nuclei
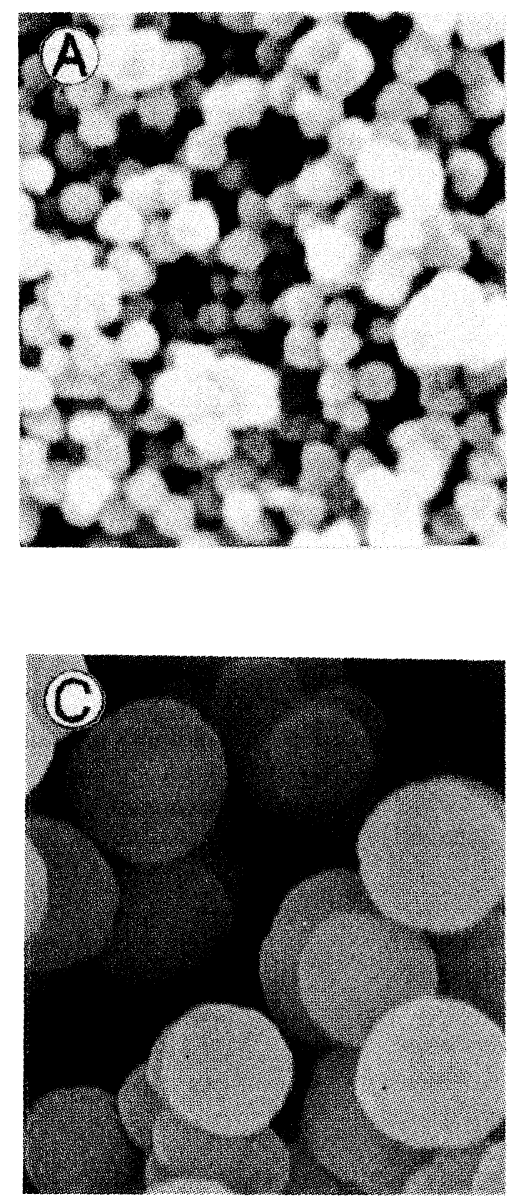

stability. The addition of water in ethanol contributed to nuclear stability.

In the ethanol-methanol mixture system,

Table II. Dependence of particle size on solvent properties

\begin{tabular}{cccc}
\hline $\begin{array}{c}\text { Fraction of } \\
\text { methanol in } \\
\text { ethanol }\end{array}$ & $\begin{array}{c}\text { Solubility parameter, } \delta \\
\end{array}$ & & Particle size \\
\hline 0.0 & 26.0 & $\mathrm{~nm}$ \\
2.5 & 26.9 & 170 \\
5.0 & 27.8 & 530 \\
7.5 & 28.8 & 1000 \\
10.0 & 29.7 & $-^{\mathrm{a}}$ \\
& & $-^{\mathrm{a}}$
\end{tabular}

${ }^{a}$ Aggregated.
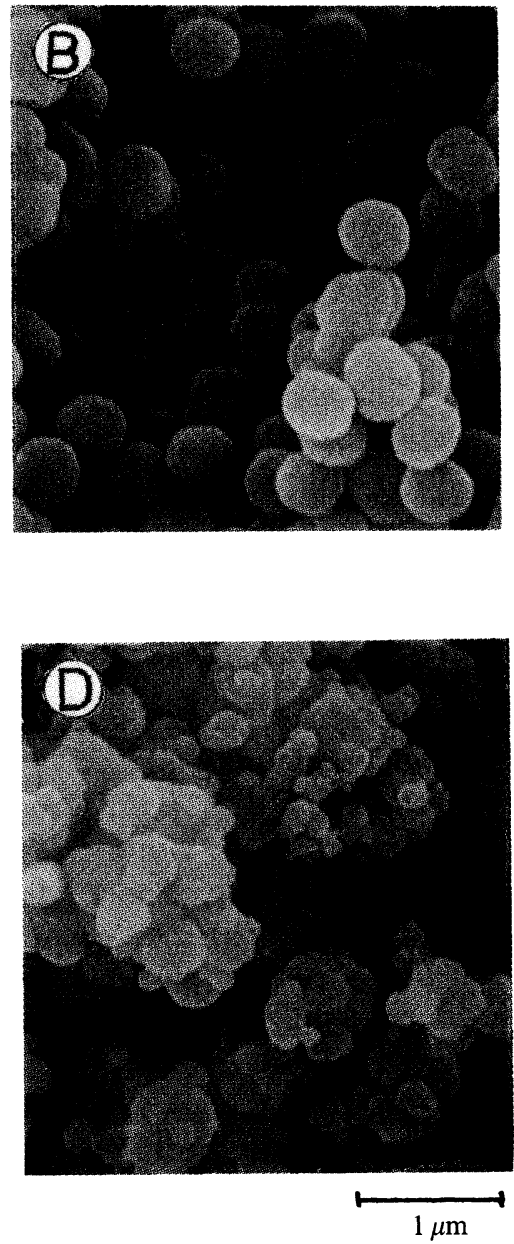

Figure 6. Scanning electron micrographs showing effects of solvent properties at AtPEGM/AAm/ MBAm $\times 10^{2} \mathrm{moll}^{-1}=0.6 / 15.4 / 4($ ethanol $/$ methanol $=\mathrm{A}, 1 / 0, \mathrm{~B}, 7.5 / 2.5, \mathrm{C}, 5 / 5, \mathrm{D}, 2.5 / 7.5)$. 

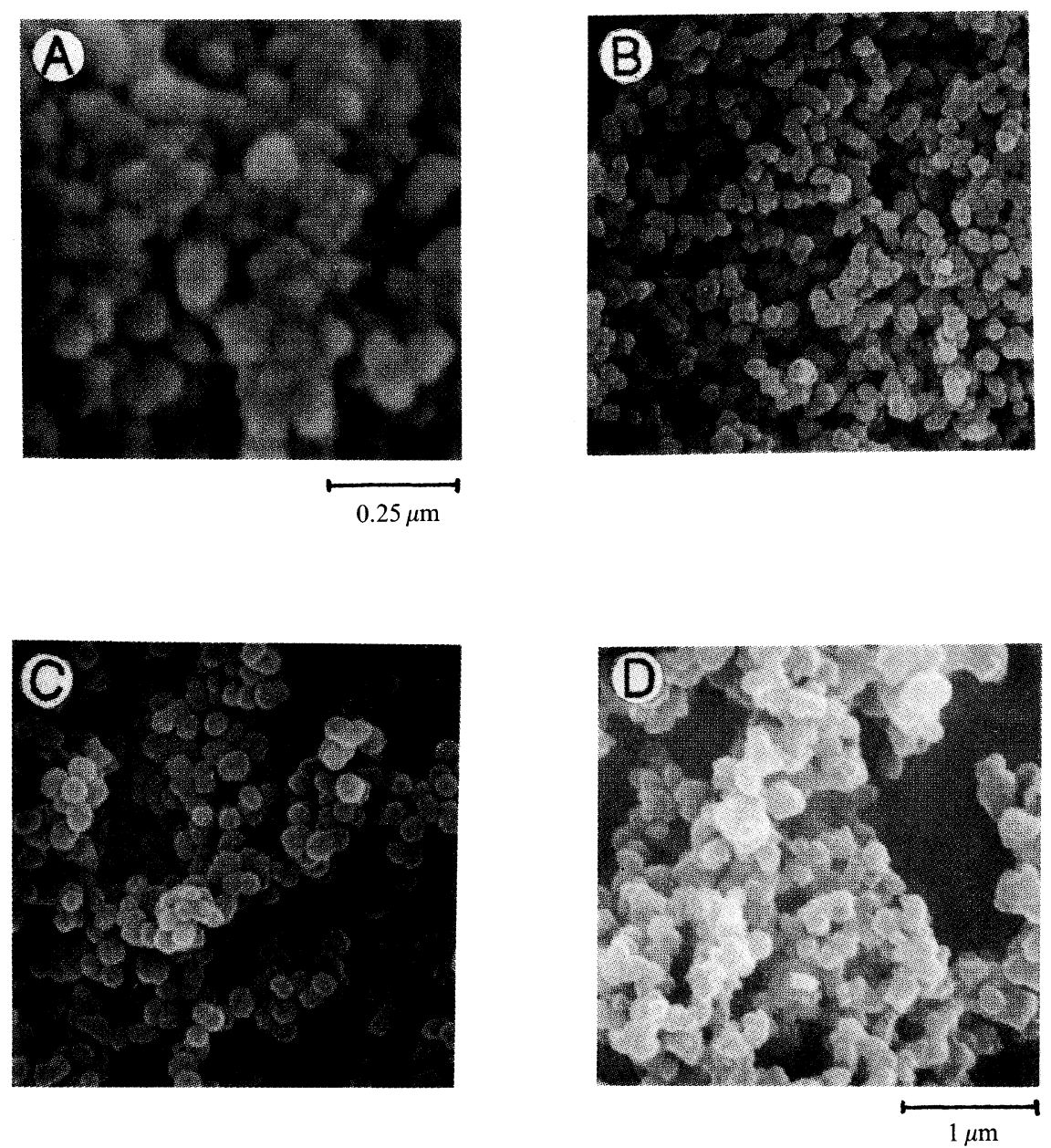

Figure 7. Scanning electron micrographs showing effects of solubility at AtPEGM/AAm/MBAm $\times$ $10^{2} \mathrm{moll}^{-1}=0.6 / 15.4 / 4$ (A, $t$-butanol, B, propanol; C, ethanol; D, methanol).

particle size increased and particle size distribution was monodisperse until a certain fraction $(0.5)$ of methanol and thereafter caused the formation of aggregates. With increasing fraction of methanol, swelling of nuclei particles increased and large particles could be prepared. But highly swollen particles might become sticky and aggregated between particles.

Table III and Figure 7 is shown the effects of solubility on particle size. From Table III, microgels of various particle sizes from $0.06 \mu \mathrm{m}$ to $1 \mu \mathrm{m}$ could be prepared by changing the nature of the solvents. The microgels were
Table III. Effects of solubility on particle size

\begin{tabular}{|c|c|c|}
\hline \multirow{2}{*}{ Solvent } & Solubility parameter, $\delta$ & Particle size \\
\hline & $\mathrm{MPa}$ & $\mathrm{nm}$ \\
\hline$t$-Buthanol & 21.7 & 60 \\
\hline Propanol & 24.3 & 130 \\
\hline Ethanol & 26.0 & 170 \\
\hline Methanol & 29.7 & ${ }^{\mathrm{a}}$ \\
\hline
\end{tabular}

${ }^{\text {a }}$ Aggregated.

monodisperse as shown in Figure 7. With increasing solubility, the degree of swelling of the precipitating nuclei particles increased and 


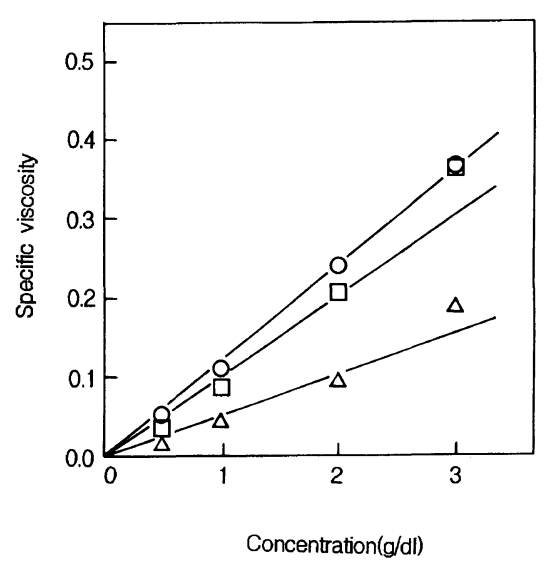

Figure 8. Specific viscosities of polyacrylamide microgels in various solvents at $25^{\circ} \mathrm{C}(O$, water; $\triangle$, ethanol; $\square$, water/ethanol $(5 / 5))$.

larger particles formed. Large size microgels formed in solvents of large solubility.

The dependence of specific viscosity with the concentrations of microgel solution was measured by the capillary method in various solvents such as ethanol and water as shown in Figure 8. The specific viscosity of a dilute dispersion of rigid spherical particles is given by $\eta_{\mathrm{sp}}=2.5 \Phi$ where $\Phi$ is concentration of microgel. Einstein provided that the slop of the curve obtained by plotting specific viscosity against particle concentration was 2.5 at lower particle concentration. ${ }^{13}$ This is called the Einstein value. But the slop of curve for polyacrylamide microgel is larger than that for hydrophobic microgel such as polystyrene and polybutene in lower concentration aqueous solution. ${ }^{14}$

The specific viscosity increased linearly at lower concentration of polyacrylamide microgels and the slope of curve obtained in water was larger than that in ethanol. This is due to relatively large value of swelling of the microgels in water.

Dispersion stability of the microgels was observed in various solvents such as ethanol, DMF, and water.

As shown in Figure 9, transmittance of the microgels in water was lower than that in DMF

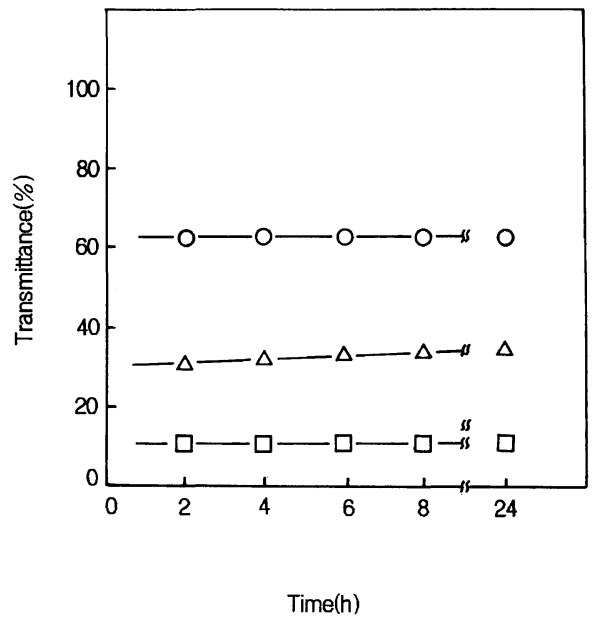

Figure 9. Stabilities of polyacrylamide microgels in various solvents ( $O$, ethanol; $\triangle$, DMF; $\square$, water).

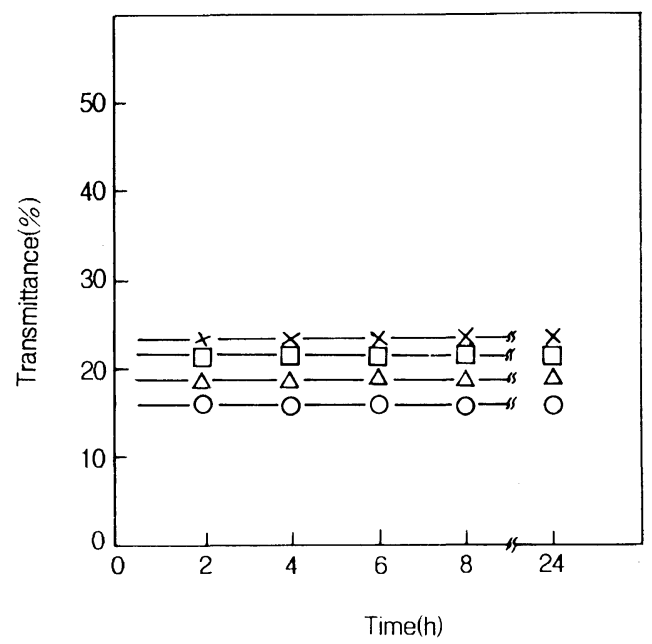

Figure 10. Effects of $\mathrm{KCl}$ concentration on polyacrylamide microgel stability (conc. of $\mathrm{KCl}\left(\mathrm{moll}^{-1}\right), \times, 0.2$; $\square, 0.15 ; \triangle, 0.10 ; \bigcirc, 0.05)$.

or in ethanol due to particle swelling. Microgels was very stable in polar solvent such as ethanol, DMF, and water.

Figure 10 shows the dependence of dispersion stability of microgels on $\mathrm{KCl}$ concentration. Microgels were very stable at various concentration of $\mathrm{KCl}$ in aqueous solution since the stability of polyacrylamide microgels supported PEG as steric stabilizer is not affected 
by salt concentration.

\section{CONCLUSION}

Microgels of $0.06-1.0 \mu \mathrm{m}$ in size were obtained by the precipitation polymerization of AtPEGM and AAm in selective solvents such as water/ethanol, ethanol/methanol, and alcoholic solvents. Microgels were stabilized by steric stabilization of AtPEGM. The nature of a solvent is the major factor determining particle size and distribution. Microgels were dispersable in water, showed very low viscosity, and were stable in polar solvents and $\mathrm{KCl}$ aqueous solution.

\section{REFERENCES}

1. T. A. Strivens and R. D. Rawling, J. Oil Colour
Chemists Assoc., 63, 412 (1980).

2. S. Wu, J. Appl. Polym. Sci., 22, 2769 (1978).

3. L. O. Kernum, Rheol. Acta, 18, 178 (1979).

4. M. Shahar, H. Meshulam, and S. Margel, J. Polym. Sci., Polym. Chem. Ed., 24, 203 (1986).

5. C. M. Klech and X. Li, J. Pharm. Sci., 79, 999 (1990).

6. R. Rembaum and W. J. Dreyer, Science, 208, 364 (1980).

7. C. K. Ober and K. P. Lok, Macromolecules, 20, 273 (1987).

8. M. R. Ferrick, J. Murtagh, and J. K. Thomas, Macromolecules, 22, 1515 (1989).

9. H. Kawaguchi, K. Fujimoto, M. Saito, T. Kawasaki, and Y. Urakami, Polym. International, 30, 225 (1993).

10. K. S. Kim, S. H. Cho, and Y. J. Kim, Polym. J., 25, 847 (1993).

11. S. Hayashi, A. Komatsu, and T. Hirai, J. Polym. Sci., Polym. Chem. Ed., 27, 157 (1989).

12. C. Price, J.-L. Forget, and C. Booth, Polym. Lett., 18, 526 (1977)

13. A. Einstein, Am. Physik., 34, 591 (1911).

14. E. S. Daniels, E. D. Sudol, and M. S. El-Aasser, Am. Chem. Soc. Symposium Series, 492, 1992, Chapter 27. 